\title{
Abirateronacetat jetzt beim Prostatakarzinom zugelassen
}

\begin{abstract}
- Anfang September hat die Europäische Kommission in einem beschleunigten Revisionsverfahren die Zulassung von Abirateronacetat (Zytiga ${ }^{\oplus}$ ) erteilt. Abirateronacetat ist der erste und einzige steroidale AndrogenBiosynthese-Inhibitor, der in Kombination mit Prednison oder Prednisolon zur Behandlung des metastasierten kastrationsresistenten Prostatakarzinoms (CRPC) bei erwachsenen Männern, deren Erkrankung während oder nach einer Docetaxel-haltigen Chemotherapie progredient verläuft, indiziert ist.
\end{abstract}

Die Zulassung basiert auf den Daten der randomisierten, doppelblinden, placebokontrollierten klinischen Pivot-Studie "COU-AA-301“. Bei insgesamt 1.195 Patienten (Abirateronacetat-Arm: $n=797$, Placebo-Arm: $n=398$ ) mit metastasiertem CRPC nach Versagen von ein bis zwei Chemotherapie-Regimen zeigte sich eine signifikante Überlegenheit der Kombination Abirateronacetat und Prednison/Prednisolon gegenüber Placebo und Prednison/ Prednisolon beim primären Endpunkt: Bereits bei der ersten, vorab geplanten Interimsanalyse betrug das mediane Gesamtüberleben 14,8 vs. 10,9 Monate (Hazard Ratio: 0,65, p < $0,001)$. Abirateronacetat senkte also das Sterberisiko um $35 \%$. Der Überlebensvorteil bestätigte sich in allen Patienten-Subgruppen, inklusive Patienten mit zwei vorherigen Chemotherapie-Regimen oder mit schlechterem ECOG-Status von 2, mit Schmerzen beziehungsweise mit radiologischer Progression zu Studienbeginn.

Die häufigsten unerwünschten Ereignisse unter Abirateronacetat waren Flüssigkeitsretention ( $31 \%$ vs. $22 \%$ ) und Hypokaliämie (17\% vs. $8 \%$ ). Diese auf dem Wirkmechanismus beruhenden unerwünschten Ereignisse waren kontrollierbar und unterschieden sich von den unerwünschten Ereignissen, die üblicherweise mit einer zytotoxischen Chemotherapie in Verbindung gebracht werden.

Nach Informationen von

Janssen-Cilag, Neuss
Harninkontinenz bei MS-Patienten: Botulinumtoxin positiv bewertet

Botulinumtoxin Typ A (BOTOX ${ }^{\circledR}$ ) hat kürzlich die Positive Opinion des Irish Medicine Boards für die Behandlung der Harninkontinenz bei Erwachsenen mit neurogener Blase erhalten, die aufgrund einer dauerhaften sub-zervikalen Rückenmarksverletzung oder Multipler Sklerose (MS) an neurogener Detrusorhyperaktivität (NDO) leiden. Bedingt durch die NDO kommt es bei diesen Patienten zu unfreiwilligen Kontraktionen der Blase während des Füllvorgangs.

Die Beurteilung des Irish Medicine Boards ist ein Schritt hin zum Erwerb der nationalen Lizenzen in den 14 europäischen Ländern, die an der „mutual recognition procedure" beteiligt sind. Die Positive Opinion ist BOTOX ${ }^{\circledR}$-spezifisch und basiert auf einem erfolgreichen weltweiten Phase-III-Studienprogramm, bei dem sich gezeigt hat, dass zielgerichtete Injektionen von Botulinumtoxin in den Blasenmuskel vorübergehend die unfreiwilligen Kontraktionen reduzieren und die Blasenkapazität erhöhen können.

Nach Informationen von

Allergan, Ettlingen

\section{Kooperationen gegen Krebs}

Künftig kooperiert das Unternehmen Lead Discovery Center (LDC) mit den deutschen Pharmakonzernen Merck und Bayer sowie mit dem kanadischen Zentrum zur Erforschung und Entwicklung von Arzneimitteln. Ziel der Zusammenarbeit ist es, die Entwicklung neuer Wirkstoffe gegen Krebs und Stoffwechselerkrankungen zu beschleunigen.

Mit Merck haben die Bio-Mediziner eine auf drei Jahre angelegte Kooperation unterzeichnet, die darauf abzielt, das Potenzial von Kinase-Hemmstoffen durch eine neuartige Herangehensweise für die zukünftige Behandlung von Krebserkrankungen besser zu nutzen.

Kinasen spielen auch bei dem Abkommen zwischen LDC und Bayer eine entscheidende Rolle: Für einen neuartigen und hochselektiven Kinase-Inhibitor erhält Bayer eine weltweite, exklusive Lizenz an den am LDC generierten Schutzrechten und dem Know-how.
Nach Informationen von

GlaxoSmithKline, München profitieren und diese den Monotherapien überlegen ist. Urologe PD Dr. Jan Lehmann,
Nach Informationen vom

Lead Discovery Center, Dortmund therapie gegenüber den Monotherapien belegen. Das unter der Kombination Tamsulosin plus Dutasterid beobachtete Profil unerwünschter Ereignisse entsprach zudem den bislang gemachten Erfahrungen mit den Einzelsubstanzen in Monotherapie. 